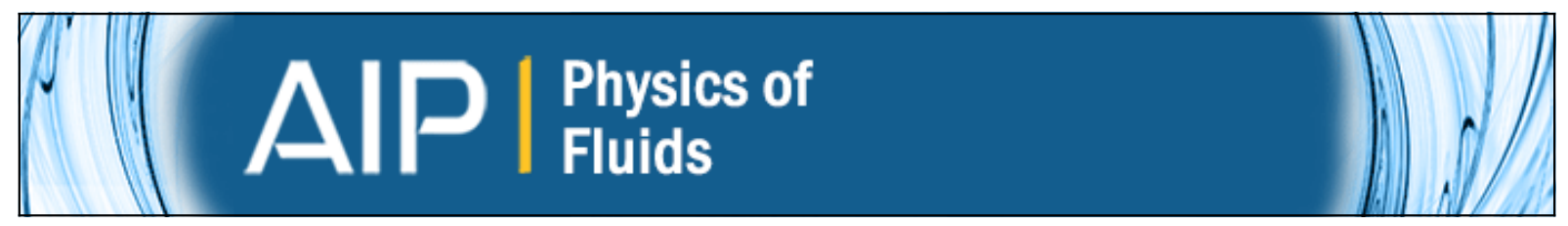

\title{
The three-dimensional interaction of a vortex pair with a wall
}

J. Alan Luton and Saad A. Ragab

Citation: Physics of Fluids (1994-present) 9, 2967 (1997); doi: 10.1063/1.869408

View online: http://dx.doi.org/10.1063/1.869408

View Table of Contents: http://scitation.aip.org/content/aip/journal/pof2/9/10?ver=pdfcov

Published by the AIP Publishing

\section{Articles you may be interested in}

The onset of three-dimensional centrifugal global modes and their nonlinear development in a recirculating flow over a flat surface

Phys. Fluids 22, 114102 (2010); 10.1063/1.3500677

Three-dimensional instabilities and transient growth of a counter-rotating vortex pair

Phys. Fluids 21, 094102 (2009); 10.1063/1.3220173

Characteristics of two-dimensional flow around a rotating circular cylinder near a plane wall

Phys. Fluids 19, 063601 (2007); 10.1063/1.2738608

On the development of three-dimensional vortex breakdown in cylindrical regions

Phys. Fluids 18, 084105 (2006); 10.1063/1.2338065

Computation of three-dimensional flows past circular cylinder of low aspect ratio

Phys. Fluids 13, 177 (2001); 10.1063/1.1332392

\section{AlP Re-register for Table of Content Alerts}




\title{
The three-dimensional interaction of a vortex pair with a wall
}

\author{
J. Alan Luton and Saad A. Ragab ${ }^{\mathrm{a})}$ \\ Department of Engineering Science and Mechanics, Virginia Polytechnic Institute and State University, \\ Blacksburg, Virginia 24061-0219
}

(Received 4 September 1996; accepted 19 June 1997)

\begin{abstract}
The interaction of vortices passing near a solid surface has been examined using direct numerical simulation. The configuration studied is a counter-rotating vortex pair approaching a wall in an otherwise quiescent fluid. The focus of these simulations is on the three-dimensional effects, of which little is known. To the authors' knowledge, this is the first three-dimensional simulation that lends support to the short-wavelength instability of the secondary vortex. It has been shown how this Crow-type instability leads to three dimensionality after the rebound of a vortex pair. The growth of the instability of the secondary vortex in the presence of the stronger primary vortex leads to the turning and intense stretching of the secondary vortex. As the instability grows the secondary vortex is bent, stretched, and wrapped around the stronger primary. During this process reconnection was observed between the two secondary vortices. Reconnection also begins between the primary and secondary vortices but the weaker secondary vortex dissipates before the primary, leaving reconnection incomplete. Evidence is presented for a new type of energy cascade based on the short-wavelength instability and the formation of continual smaller vortices at the wall. Ultimately the secondary vortex is destroyed by stretching and dissipation leaving the primary vortex with a permanently distorted shape but relatively unaffected strength compared to an isolated vortex. (C) 1997 American Institute of Physics. [S1070-6631(97)03110-3]
\end{abstract}

\section{INTRODUCTION}

The interaction of vortices with surfaces is an important phenomenon in many engineering applications. For instance, trailing vortices from aircraft which interact with the ground can present a danger to following aircraft. ${ }^{1}$ In assessing the danger it is important to understand the motion, structure, and decay of the vortices. These characteristics could be strongly influenced by three-dimensional effects. Vortices shed from the aircraft body and leading edge extension of delta wings pass over the wing thereby affecting the lift, drag, and possible control of the aircraft. Similarly in separating flows, vortical structures can form which later interact with the solid surface downstream of reattachment. ${ }^{2,3}$ These vortical structures can induce secondary separation and pressure fluctuations along the solid surface. Also, in helicopter aerodynamics the wakes from upstream blades can interact with blades downstream, possibly leading to undesirable vibrations. $^{4,5}$

There are many studies which focus on either vortex rings or pairs impinging on a no-slip wall in an otherwise stagnant fluid. A recent review of vortex/wall interactions can be found in Doligalski et al. ${ }^{6}$ A vortex moving toward a wall is often observed to reverse course and move away from the wall. This movement is known as vortex rebound and has been observed in free-flight studies for wing tip vortices near the ground (see, for instance, Dee and Nicholas ${ }^{7}$ ). An explanation for the rebound phenomenon was given by Harvey and Perry ${ }^{8}$ who experimentally studied the motion of a single wing tip vortex near a moving wall. The primary vortex creates a layer of vorticity of opposite sense next to the wall.

\footnotetext{
${ }^{a}$ Corresponding author. Telephone: (540) 231-5950; Electronic mail: ragab@ragab1.esm.vt.edu
}

This vortex sheet becomes unstable, separates, and rolls up to form a secondary vortex. The secondary vortex induces an upward motion to the primary vortex in an inviscid-like fashion. Boldes and Ferreri ${ }^{9}$ examined a vortex ring approaching a wall. The ring was created by a drop of colored water impacting the free surface of a quiescent body of water. They reported the rebound of the vortex from the wall, and in some cases, multiple rebounds. In their two-dimensional Navier-Stokes calculations, Peace and Riley ${ }^{10}$ demonstrated the rebounding phenomenon of a vortex pair from a no-slip boundary. Their simulations were for low Reynolds number (up to $\operatorname{Re}_{\Gamma}=\Gamma / \nu=150$ ). Flow separation was not observed. In their detailed experimental study of vortex rings, Walker et al. $^{11}$ (also see Cerra and Smith ${ }^{12}$ for related earlier work) created vortex rings by the sudden ejection of fluid through a sharp edged orifice. As the ring approached a wall, the formation of secondary and tertiary vortex rings was observed. The secondary ring moves around the primary ring and into its center. The secondary ring not only causes the primary ring to rebound, but arrests the radial expansion of the ring. For a sufficiently strong vortex, the diameter of the primary ring will shrink, a process known as reversal. As the secondary ring moves into the center of the primary ring, azimuthal waves develop on the secondary ring, but not the primary ring. Walker et al. showed that these fluctuations are associated with the compression of the secondary ring. They also observed the ejection of the secondary vortex ring from the center of the primary ring. Orlandi, ${ }^{13}$ in his two-dimensional Navier-Stokes simulations, observed the formation of secondary and tertiary vortices for a vortex pair impacting a no-slip wall. His simulations were for Reynolds number up to $\operatorname{Re}_{\Gamma}=3200$. He also observed the formation of a secondary vortex pair that moved far from the wall, similar to the secondary ring ejection in the experiments of Walker et al. 
In a subsequent study Orlandi and Verzicco ${ }^{14}$ observed the multiple formation of vortices for the case of a vortex ring. They concluded that vortex pairing was the mechanism for the ejection of the secondary ring from the center of the primary ring. The results also showed the growth of azimuthal instabilities in the secondary ring, similar to the experimental results of Walker et al. The stability of the secondary vortex ring was investigated by Swearingen et al. ${ }^{15}$ using numerical simulations and a localized stability analysis. In the simulations the secondary vortex ring developed azimuthal perturbations similar to those seen by Walker et al., ${ }^{11}$ Orlandi and Verzicco, ${ }^{14}$ and others. The stability analysis followed the approach of Widnall and Sullivan ${ }^{16}$ who showed that an isolated vortex ring is stable to longwavelength disturbances (but unstable to short-wavelength ones). However, the analysis of Swearingen et al. showed that the presence of another vortex ring causes the longwavelength disturbances to grow. Their analysis gave results in good agreement with the simulations in the early stages of development of the instability.

Most numerical studies of vortex pairs near a wall have been two-dimensional. However, in three dimensions the pair is subject to instabilities, in particular, those named after Crow. Crow ${ }^{17}$ modeled a pair of trailing vortices as sinusoidally perturbed vortex filaments. The mode shape is a sinusoidal perturbation of each filament that is confined to a plane inclined at approximately $45^{\circ}$ to the plane in which the undisturbed vortices lie. The configuration is symmetric about a plane that bisects the distance between the vortices. The analysis predicts two groups of unstable waves with one set having long wavelengths and the other having short wavelengths (comparable to the core diameter). Crow gives the maximum dimensionless growth rate $\left(\bar{\alpha}=2 \pi b^{2} \alpha / \Gamma\right)$ as 0.8 for the long waves and 1.0 for the short waves. Here $b$ is the distance between the vortices and $\Gamma$ is the circulation of each vortex. Widnall et al. ${ }^{18}$ showed that the shortwavelength instability found by Crow is spurious since its predicted wavelength violates the assumptions of the model. The analysis of Widnall et al. is valid for slender vortices $(a / b \ll 1$, where $a$ is the core diameter) and $k a=O(1)$, where $k$ is the wavenumber. This analysis is therefore appropriate for short-wavelength disturbances, as opposed to the analysis of Crow which requires $k a \ll 1$. Although the shortwavelength instability predicted by Crow does not exist, Widnall et al. showed that for vortices of finite core size there exists higher radial bending modes which are unstable. Recently Thomas and Auerbach ${ }^{19}$ observed the formation of both the long- and short-wavelength instabilities for a vortex pair. The pair was composed of the starting and stopping vortices that formed on the edge of a rotated plate. Both long and short-wavelength instabilities have been seen in the current simulations.

The disturbances grow until portions of each vortex come into close contact with one another. At these points vortex reconnection occurs which transforms the pair into a series of rings. While vortex reconnection has received much attention, especially in recent years, it is still a poorly understood phenomenon. A central concept is the dissipation (or "cancellation") of vorticity in regions where antiparallel vorticity lines are close together. The vortex lines then undergo a cross linking, or bridging, process which connects the two vortices. The cross linked vorticity is amplified by vorticity stretching. Common configurations for the study of vortex reconnection are the collision of two vortex rings ${ }^{20,21}$ and a sinusoidally perturbed vortex pair. ${ }^{22,23}$ An extensive discussion of the reconnection process is given by Kida and Takaoka. ${ }^{24}$

Recently Dommermuth presented three-dimensional simulations of vortex pairs interacting with free-slip and noslip walls ${ }^{25}$ as well as free surfaces. ${ }^{26}$ The primary motivation for this work was to understand the formation of free surface features such as striations and scars that can form when a rising vortex pair approaches a free surface. These features were observed in the experiments of Sarpkaya and Suthon $^{27}$ and Sarpkaya. ${ }^{28}$ Since Dommermuth was concerned with the reconnection process and not the mechanism by which the primary vortex is deformed, a sinusoidal perturbation was initially imposed on the core position of the vortex. Sheets of helical vorticity were observed spiraling off of the vortex before interaction with the surface. The author suggests that the vorticity sheets originated from an inviscid instability caused by large changes in the curvature of the vortex along its axis. The helical vorticity sheets evolved into "beads" of cross-axis vorticity as they revolved around the primary vortex. For no-slip wall interactions, the helical vorticity sheets merged with the secondary vorticity sheet at the wall to form U-shaped vortices wrapped around the primary vortex. For free surface interactions, the cross-axis vorticity reconnected at the free surface. The cross-axis vortices appear to be responsible for the formation of the striations and scars observed in the experiments.

In Dommermuth's work the axial length of the computational domain was chosen to be too short to permit the long-wavelength Crow instability to form. The shortwavelength Crow-Widnall instability was not addressed. In the current work the Crow-Widnall instability and the interaction between the primary and secondary vortices are key features of the flowfield. Instead of an initial sinusoidal disturbance of the primary vortex core a random disturbance is used which allows any instabilities present to develop naturally. Because of this form of the initial disturbance the helical sheets of vorticity which played a fundamental role in the vorticity dynamics of Dommermuth's simulations are not present. The instabilities seen in the current work occur after the primary vortex interacts with the wall and the subsequent formation of a secondary vortex.

In the current study we consider the case of a counterrotating vortex pair approaching a solid surface. Our object is to reveal the three-dimensional characteristics of the interaction of the pair of vortices with the wall. The full threedimensional, unsteady, incompressible Navier-Stokes equations are solved. In Section II the numerical scheme is outlined as well as initial and boundary conditions. The results are presented in Section III while the conclusions are discussed in Section IV. 


\section{METHOD OF SOLUTION}

\section{A. Numerical scheme} by

The incompressible Navier-Stokes equations are given

$$
\begin{aligned}
& \frac{\partial u_{i}}{\partial t}+\frac{\partial}{\partial x_{j}} u_{i} u_{j}=-\frac{\partial p}{\partial x_{i}}+\frac{1}{\operatorname{Re}} \frac{\partial}{\partial x_{j}} \frac{\partial}{\partial x_{j}} u_{i}, \\
& \frac{\partial u_{i}}{\partial x_{i}}=0,
\end{aligned}
$$

where all variables have been nondimensionalized by a characteristic velocity and length. The equations are solved by a scheme first proposed by Kim and $\mathrm{Moin}^{29}$ and later modified by Le and Moin. ${ }^{30}$ The scheme is described in detail in these references so a mere overview is given herein. The scheme consists of a fractional-step (time-splitting) method combined with the approximate factorization technique. The momentum equation is advanced in time in two steps, first applying the convection and diffusion operators and then the pressure operator. Finding the pressure consists of solving Poisson's equation, which is equivalent to satisfying the continuity equation. Solving Poisson's equation is by far the most computationally expensive step. Le and Moin proposed a modification to the Kim and Moin method to increase the CFL while reducing the computational effort. Their method employs a three-stage Runge-Kutta scheme in which the convective terms are advanced explicitly and the viscous terms implicitly. The stability limit is CFL $=\sqrt{3}$ for the onedimensional convection-diffusion equation. The Kim and Moin time splitting is applied at each stage, yet the Le and Moin modification permits Poisson's equation to be solved only at the end of the time step, rather than at each of the three stages. This results in a substantial reduction in the CPU time. Le and Moin estimated the CPU time savings to be $68 \%$ over the Kim and Moin scheme for their simulations of flow over a backward facing step. In the current work a multigrid method is used to solve Poisson's equation. This implementation of the multigrid method uses Gauss-Seidel line relaxation and semi-coarsening on uniform or stretched meshes. The scheme provides a very efficient solution to Poisson's equation which is critical to reducing the computational time. The accuracy of the code has been thoroughly examined by Luton. ${ }^{31}$

\section{B. Initial and boundary conditions}

Now we consider the initial and boundary conditions for a vortex pair approaching a wall. The computational domain is shown in Fig. 1. The two primary vortices are initially parallel to the $z$-axis and extend from $z=-\infty$ to $z=+\infty$. The $x=0$ plane bisects the distance between the vortices and is assumed to be a plane of symmetry throughout the simulation. For the boundary conditions, which are only needed for the velocity, the no-slip and no-penetration conditions are applied at the wall $(y=0$ plane). Due to symmetry only one vortex of the pair is simulated. Thus symmetry boundary conditions are applied along the center $(x=0)$ plane of the vortex pair. Symmetry conditions are also specified on the boundaries opposite the wall (top) and opposite the center

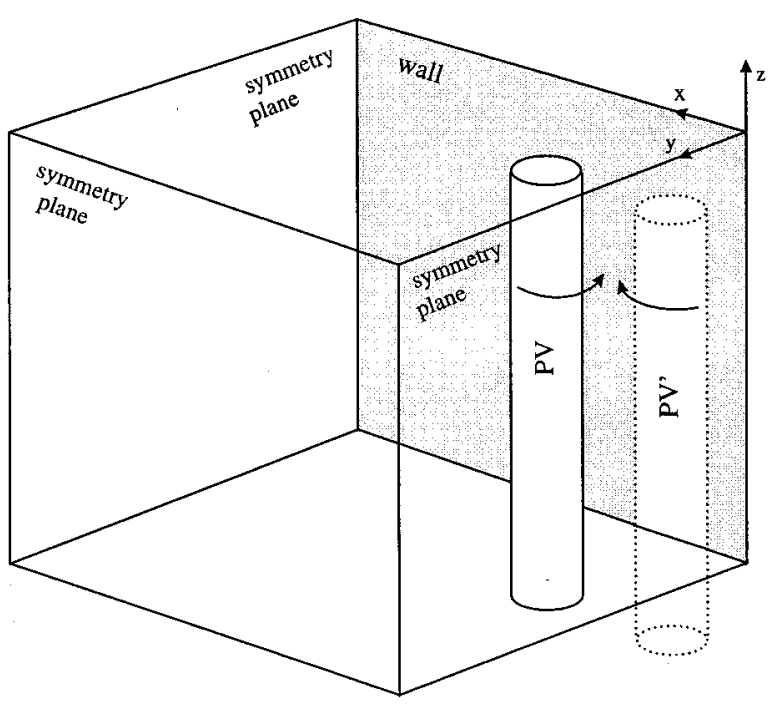

FIG. 1. The computational domain.

plane (right). These boundaries are much further away and do not affect the flowfield substantially as will be discussed in Section III D (also see Luton ${ }^{31}$ ). Periodic conditions are used in the axial $(z)$ direction. The velocity field is initialized using a Lamb-Oseen vortex which has a velocity profile given by

$$
v_{\theta}=\frac{A v_{c}}{r / r_{c}}\left[1-e^{-B r^{2} / r_{c}^{2}}\right]
$$

where $v_{\theta}$ is the tangential velocity, $v_{c}$ is the maximum tangential velocity, $r$ is the distance in the radial direction, $r_{c}$ is the core radius, and $A$ and $B$ are constants given by $A$ $=1.39795$ and $B=1.25643$.

The vortex images across the center plane and wall are included as well. The images across the other two symmetry boundaries are also taken into account, but since they are further away they have little effect. While these initial conditions are divergence free and satisfy the no-penetration boundary condition on the wall, they violate the no-slip condition. However, the vortex is sufficiently far away from the wall that the resulting disturbance is negligible.

In addition to this basic state, a random disturbance is added to the initial velocity field at all points in the computation domain. All possible modes are excited randomly to ensure that all, if any, instabilities of the flow are affected. The disturbance is subject to certain restrictions. The first is that the disturbance must have a periodicity of $L_{z}$ (the length of the domain in the axial direction) in order to be compatible with the boundary conditions. Thus the form of $x$-component of the disturbance velocity is taken to be

$$
u^{\prime}(x, y, z)=\operatorname{Real}\left[\sum_{m=1}^{N_{z} / 2} \hat{u}_{m}(x, y) e^{i k z}\right],
$$

where $\hat{u}_{m}$ is the complex amplitude, $k=2 \pi m / L_{z}$ is the wave number, $N_{z}$ is the number of points in the $z$-direction, $m$ is the mode number, and $i=\sqrt{-1}$. The other velocity components have a similar form. Since all incompressible flows must have a divergence free velocity field the amplitudes 
cannot be specified independently. If $\hat{u}_{m}$ and $\hat{v}_{m}$ are given then $\hat{w}_{m}$ must be found from the discrete form of the continuity equation. Two methods have been used to determine $\hat{u}_{m}$ and $\hat{v}_{m}$. Both rely on calls to a random number generator. The first method, denoted here as Rand, assigns random numbers between $-1 / 2$ and $1 / 2$ to the real and imaginary parts of the complex amplitude. This is done at every point in the cross $(x y)$ plane and for every mode number $m$. Thus the only structure to the disturbance field is that it is periodic in $z$. The second method, denoted by RandB, assigns random numbers in the range of 0 to 1 . Consequently the velocity field has some structure in the cross plane, while the Rand disturbance does not. The RandB disturbance is more representative of a flow that is being forced. For instance, let us consider the trailing vortex of a wing. Time varying perturbations could be introduced at the wing tip. These disturbances would be wrapped up into the vortex and create variations along its axis. This has been done experimentally ${ }^{32}$ and during flight ${ }^{33}$ to excite the Crow instability between trailing vortices. The current simulations, however, are applicable to instabilities that occur between a trailing vortex and the secondary vortex that is created as the trailing vortex interacts with the ground. The RandB disturbance could give one an idea as to the potential of affecting the long time characteristics of the flow by forcing.

A variation of the RandB disturbance, denoted by RandC, was also used. This disturbance is identical to RandB except that $\hat{u}_{m}=\hat{v}_{m}$. Thus the cross plane velocity lies in the directions defined by the line that is $45^{\circ}$ from the positive $x$ and $y$-axes. This was used in order to more strongly excite the Crow instability.

\section{RESULTS}

\section{A. The long-wavelength Crow instability}

As an introduction to some of the three-dimensional effects of a vortex pair impinging on a wall we shall briefly examine one type of instability exhibited by an isolated vortex pair. The instability is named after Crow who laid the theoretical foundation. ${ }^{17}$ The initial and boundary conditions are the same as those in Section II B with the exception that the wall is replaced with symmetry conditions. Thus the boundaries parallel to the vortex axis are in conflict with the configuration as given by Crow for an isolated vortex pair. The boundary conditions were selected so that a small domain could be used. Thus the vortex follows a circuit along the inside edges of the domain instead of traveling long distances in a straight line (as an isolated pair would). In any case, we are not concerned here with a detailed analysis of the Crow instability but merely a demonstration of its properties.

Let us first consider a vortex initially located at $x=2$, $y=5$ with a Reynolds number based on the circulation of $\operatorname{Re}_{\Gamma}=8784$. In the cross plane the domain extends from $x$ $=0$ to $x=10$ and $y=0$ to $y=10$. The length of the domain in the $z$-direction is 28 . All quantities have been nondimensionalized by the initial core radius, $r_{c}$, and maximum tangential velocity, $v_{c}$. The grid is uniform with a size of $64 \times 64$ $\times 32$. The vortex has positive vorticity and therefore initially

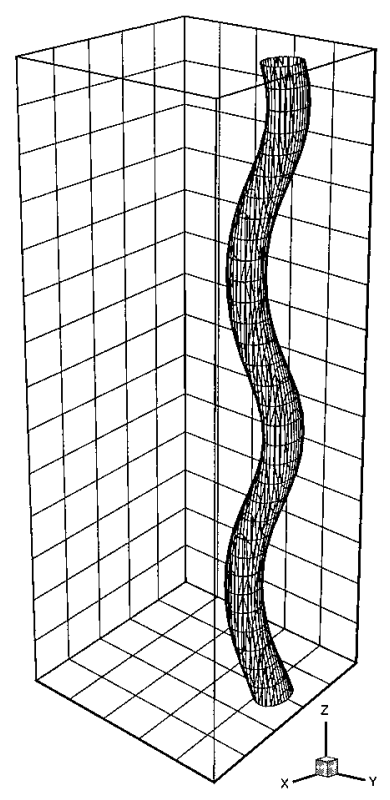

(a)

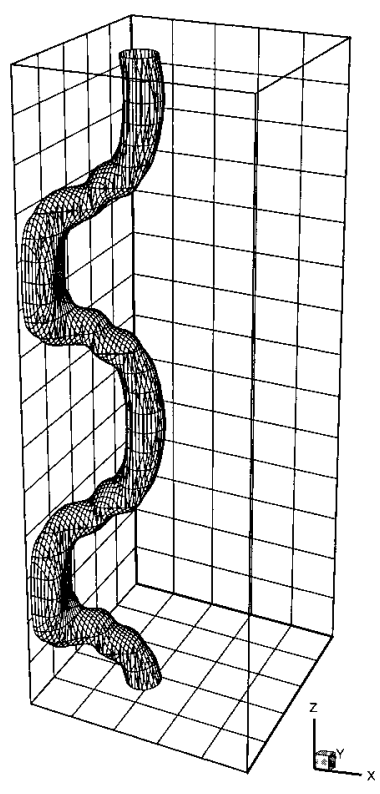

(b)
FIG. 2. Surfaces of constant vorticity $\Omega=1.0$. (a) $t=167$, (b) $t=196$.

moves in the negative $y$-direction due to the influence of its image across the symmetry plane at $x=0$. A RandB disturbance of magnitude $U_{\mathrm{rms}}^{\prime}=0.04 v_{c}$ was added to the initial velocity field. Only the second mode $\left(\lambda_{z}=14\right)$ was excited.

A surface of constant vorticity at time $t=167$ reveals the undulations of the vortex that have arisen due to the longwavelength Crow instability [Fig. 2(a)]. The disturbance continues to grow until parts of the vortex approach a symmetry plane, that is, the image vortex. The process of reconnection then begins which transforms the vortex pair into a series of rings. In Fig. 2(b) the vortex is beginning to reconnect as evident by the formation of half-ring structures-the other halves are the images. After this point in time the corner of the domain begins to significantly alter the flow. Nevertheless, the simulation illustrates the nature of the Crow instability. The characteristic $45^{\circ}$ angle of the Crow instability can be seen by projecting the isovorticity surface onto an $x y$-plane. This has been done at time $t=167$ (Fig. 3). The vortex is moving in the clockwise direction. The slight bend at one end is most likely due to the interaction at the corners.

The wavelength of the instability seen in the previous simulation is $14 r_{c}$. However, for this vortex pair Crow predicts that the most unstable wave has a wavelength of $21 r_{c}$. In order to compare with the theory another simulation was run with a $64 \times 64 \times 8$ grid and $L_{z}=21$. Only the first mode $\left(\lambda_{z}=L_{z}\right)$ was excited with a RandC disturbance of $U_{\mathrm{rms}}^{\prime}$ $=0.20 v_{c}$. The growth rate was found by taking a fast Fourier transform in the $z$-direction of the total kinetic energy. After an initial decay the energy in the first mode grows exponentially at a rate of 0.071 . When nondimensionalized as defined by Crow the growth rate is $\bar{\alpha}=0.81$. The growth rate predicted by Crow for this mode is $\bar{\alpha}=0.80$. This compares very well, especially considering the very coarse grid in the $z$-direction. Later in time the corners of the domain cause interactions that alter the growth rate. A systematic 


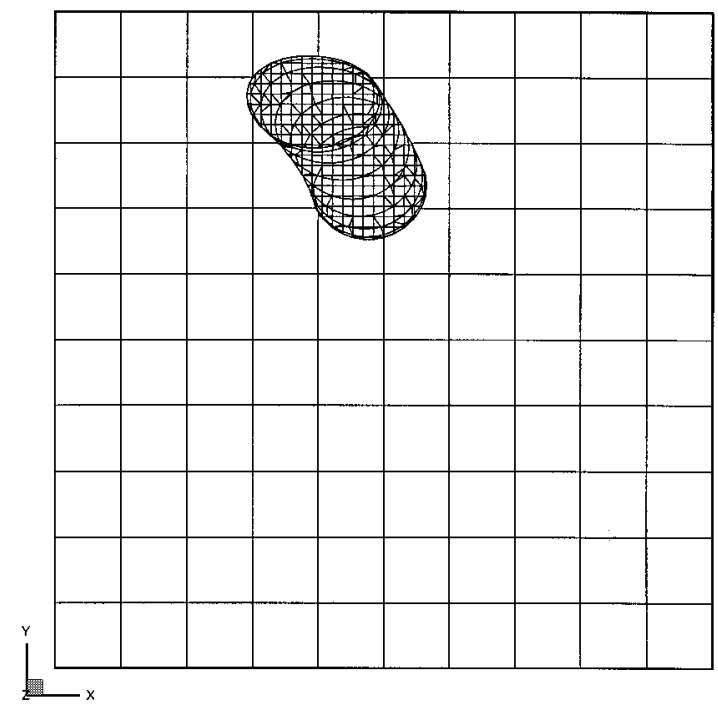

FIG. 3. Projection of an isovorticity surface onto an $x y$-plane. $t=167, \Omega$ $=1.0$.

study of the Crow instability would require more appropriate boundary conditions. The features illustrated here, however, are ones that will be seen in simulations of a vortex pair impinging on wall.

\section{B. The strucutre of the short-wavelength instability}

Let us now turn our attention to the problem of a vortex pair impinging on a wall. The initial and boundary conditions are the same as those discussed in Section II B. The vortex is initially located at $x=2, y=5$ and has a Reynolds number of $\operatorname{Re}_{\Gamma}=2196$. The size of the domain is $L_{x}=L_{y}$ $=10$ and $L_{z}=4$. A uniform grid of size $96 \times 96 \times 48$ is used resulting in 19 grid points in the vortex core. The reason for choosing $L_{z}=4$ will become apparent later. In order to excite all possible modes of instability a Rand-type disturbance is used with $U_{\mathrm{rms}}^{\prime}=0.20 v_{c}$. Only the first mode $\left(\lambda_{z}=L_{z}\right)$ is excited. Simulations which were run without an initial random disturbance showed three dimensionality after long times.

Let us first turn our attention to the distribution of vorticity on horizontal planes. In Fig. 4 are shown contour plots of $\omega_{z}$ in the mid and top planes at time $t=43.2$. In the mid plane the cross sectional shape of the secondary vortex is noncircular-more so than the primary. Between the opposite signed vortices a large vorticity gradient exists. Consequently this is a high dissipation region. In the top plane the vortices are further apart showing that the flow has become three dimensional. It is also apparent that the structure of the secondary vortex (SV) varies in the $z$-direction depending on its proximity to the primary vortex (PV). The "offset" of the vortex center indicates that this is not the first radial mode but a higher one. For the first radial mode the vortex core bends as one unit whereas the second radial mode has a more complex structure. ${ }^{18}$

In Fig. 5 are shown the trajectories of each vortex for a similar simulation on a coarser grid $(64 \times 64 \times 16)$. While the path of the primary vortex is very similar to what is seen in

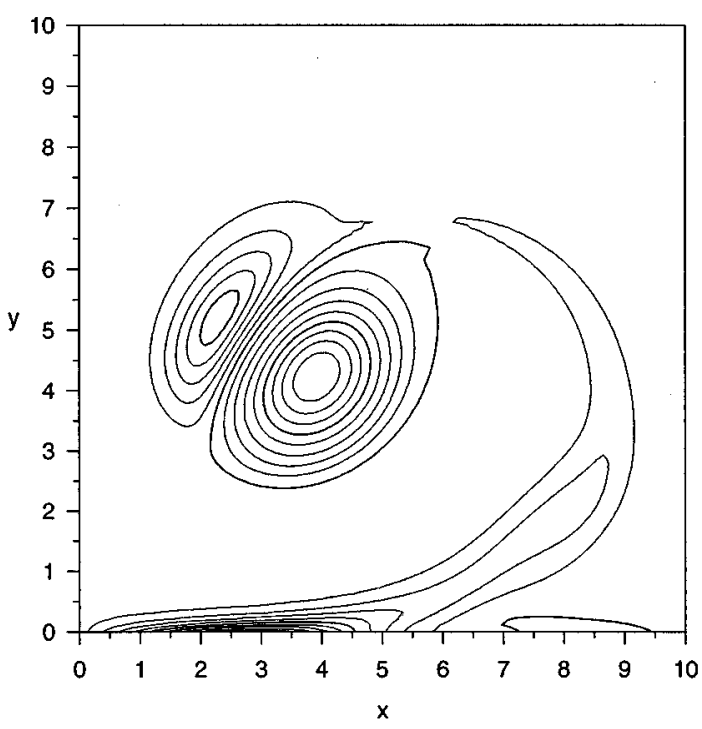

(a)

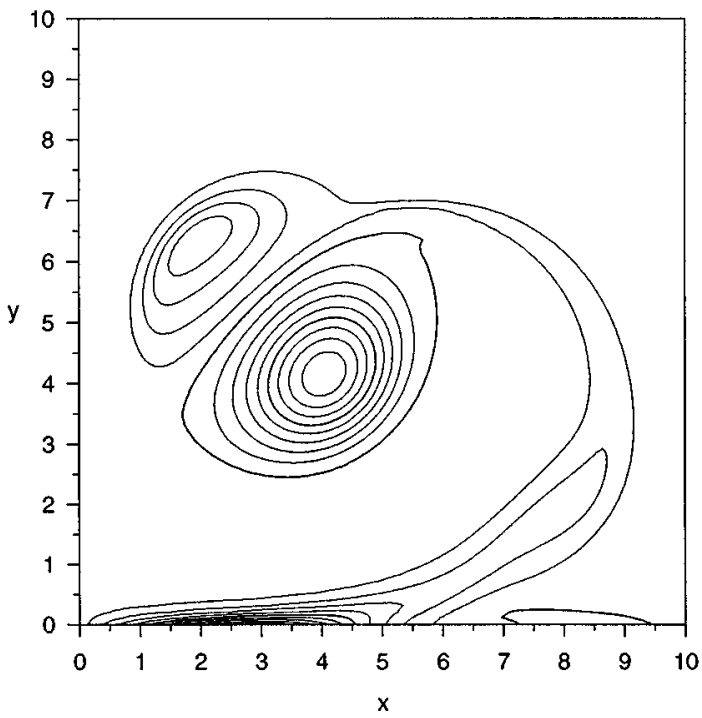

(b)

FIG. 4. Vorticity contours of $\omega_{z}$ on two different horizontal planes at $t$ $=43.2$. The contour levels range from -2 to 2 by 0.2 . (a) Mid plane, (b) top plane.

two-dimensional studies, the trajectory of the secondary vortex is dramatically different. Two curves are shown for the $\mathrm{SV}$ corresponding to the mid plane $\left(\mathrm{SV}_{\mathrm{mp}}\right)$ and top plane $\left(\mathrm{SV}_{\mathrm{tp}}\right)$ of the domain. Shortly after the SV forms the curves are coincident indicating that (most likely) the SV is straight in the $z$-direction. Before long, however, the curves begin to diverge. The symbols on the curves allow one to see the relative position of the vortices. For the position of the vortices marked by the last two sets of symbols, the angle of the $\mathrm{SV}$ to the $\mathrm{SV}_{\mathrm{mp}}-\mathrm{PV}$ line is approximately $49^{\circ}$. This is rather suggestive since the angle predicted by Crow is approximately $45^{\circ}$. However, this situation is different in that the vortices are of unequal strength and are close together. Instead of the symmetric Crow-type instability the SV is distorted by the strong PV but the weaker SV is unable to significantly bend the PV.

A time series of isovorticity surfaces is shown in Fig. 6. 


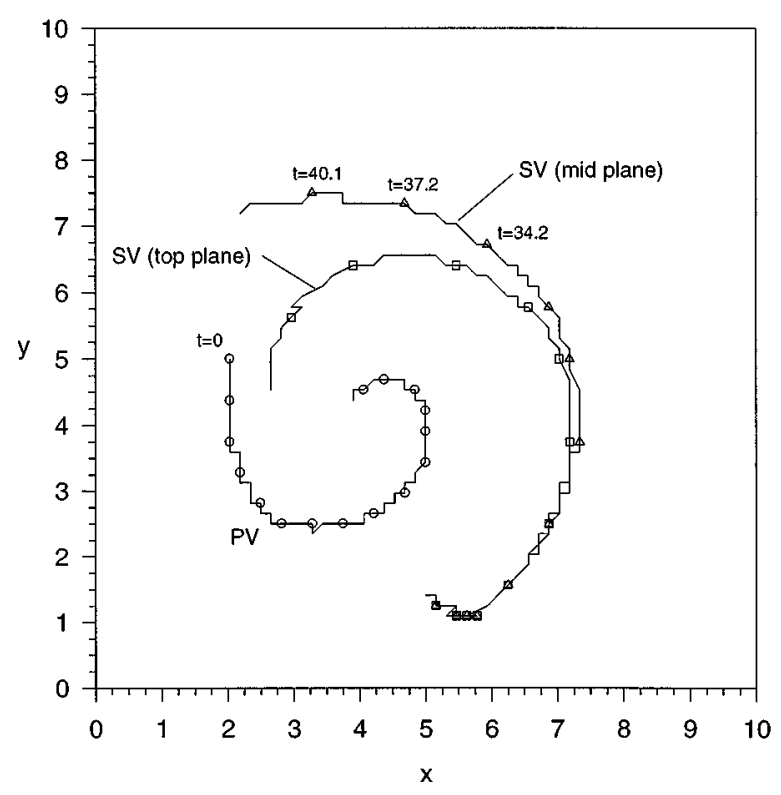

FIG. 5. The trajectories of the vortices.

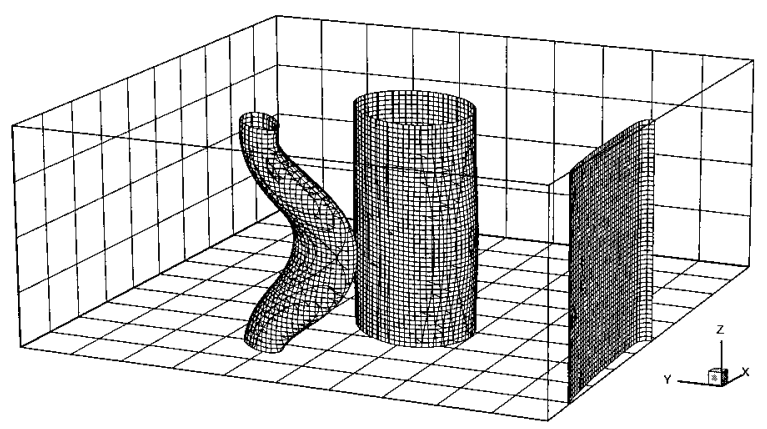

(a)

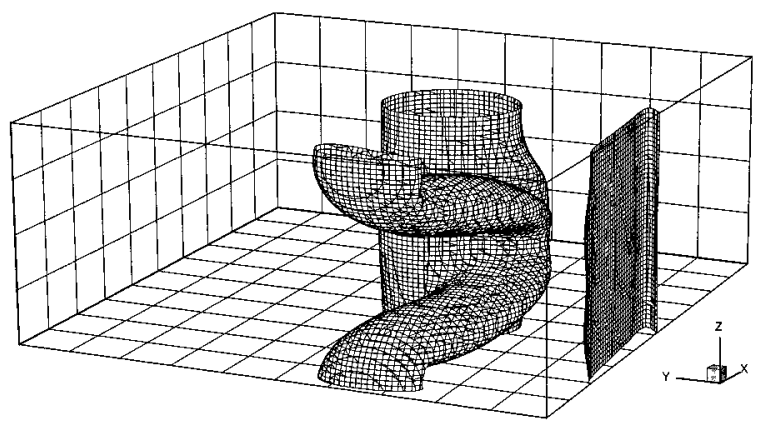

(b)
At time $t=43.2$ the secondary vortex has already formed and completed one half of a circuit around the primary vortex. It is about to enter the region between the PV and the center symmetry plane. The SV is already bent significantly yet the $\mathrm{PV}$ remains straight. The cross sectional diameter of the SV is no longer constant but is larger in the region closest to the $\mathrm{PV}$. As the SV moves toward the wall its instability grows very rapidly. By time $t=50.9$ the SV has assumed a hairpin shape. We shall refer to the two horizontal portions of the $\mathrm{SV}$ as "legs" and the vertical portion near the PV as the "head." Due to the periodic boundary conditions there is half of a head at the top and bottom of the domain near the center plane. These heads have moved toward the center plane and become highly elongated in the cross plane due to the close proximity of the image. The central section of the SV is very close to the PV. A hump on the isovorticity surface appears on the PV in this region. This is better seen in Fig. 6(c) which is a view in the opposite direction at $t$ $=57.3$. At this vorticity level the isovorticity surfaces of the secondary and primary vortices have merged in the region of closest contact. This region has a strong vorticity gradient

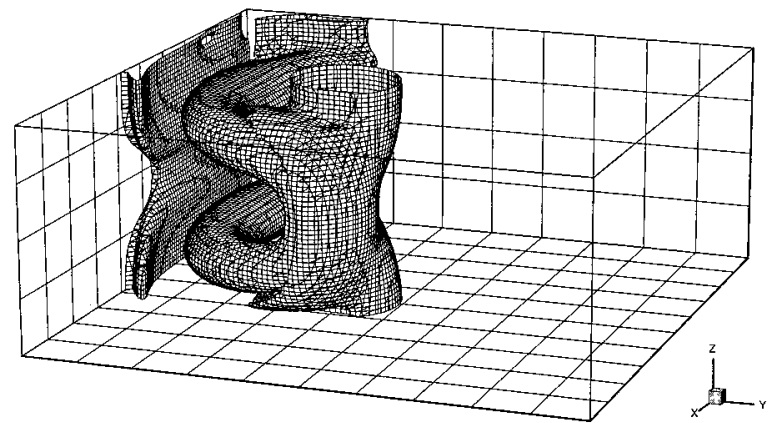

(c)

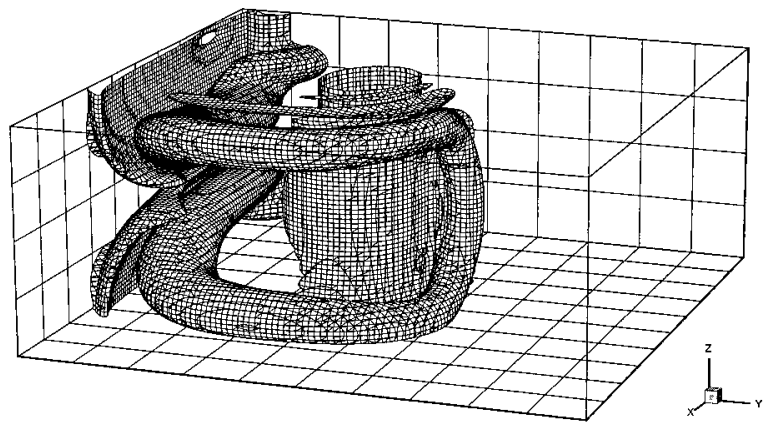

(d)

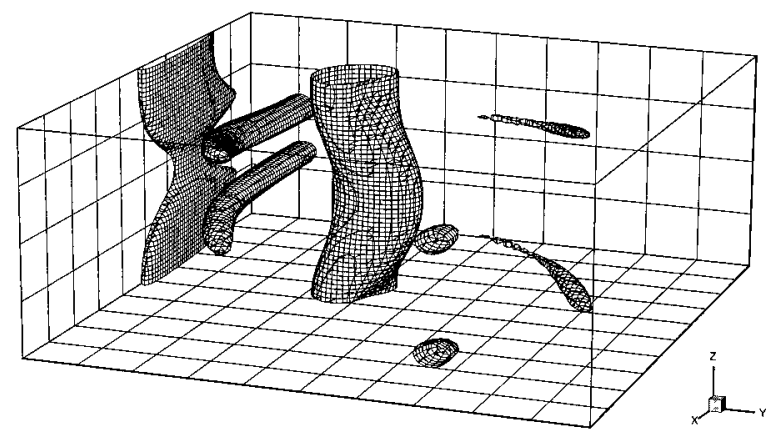

(e)

FIG. 6. Surfaces of constant vorticity magnitude $\Omega=0.7$. (a) $t=43.2$, (b) $t=50.9$, (c) $t=57.3$, (d) $t=64.8$, (e) $t=100.0$. 
and therefore high dissipation. As the axial vorticity is dissipated the remaining vorticity lines of each vortex can link with one another thus "bridging" the gap. This is the general process by which reconnection occurs. The reconnection region is therefore known as a "bridge." ${ }^{24}$ Although it is unclear whether any bridging has taken place, the PV is showing significant deformation now. At the center plane the SV continues to distort. Only a thin region extends in the $z$-direction. Reconnection here appears imminent.

The legs of the SV now lie almost entirely in the horizontal plane and have moved close to the wall. As with any vortex that approaches a wall, opposite signed vorticity is created at the surface. The wall vorticity layer can be seen in the early stages of formation in Fig. 6(c). The sign of vorticity in the SV is such that the legs induce a flow near the wall that is in the positive $y$-direction between the legs. At the top and bottom of the domain the flow is toward the wall. The vorticity layer induced by the SV has begun to lift away from the wall. This is more apparent at time $t=64.8$. Each uplifted layer created by a SV leg can roll up to form an even smaller tertiary vortex (TV). It is likely that viscous effects prevent this from happening in this simulation. At higher Reynolds numbers tertiary vortices would form from the uplifted vorticity layers. Also the SV has now wrapped even further around the PV. This stretches the secondary vortex resulting in a smaller cross sectional diameter. At the center symmetry plane a portion of the SV has connected with its image. This event will be discussed in more detail shortly.

In order to gain insight into the fate of the vortices the vorticity distribution at $t=100.0$ is presented in Fig. 6(e). At this large time numerical dissipation might have some effect in redistributing the vorticity. In any case we can draw some important conclusions from the general features of the flow. Foremost is the fact that the primary vortex has remained strong while the SV has been nearly destroyed by the stretching due to the PV and viscous dissipation. Two weak portions of the SV remain near the wall. At the center plane the legs have finished reconnecting. Reconnection between the primary and secondary vortices, however, did not occur. Soon only the primary vortex will remain. The eventual outcome is similar to the initial flow but the primary vortex retains a permanent distortion due to its interaction with the wall.

Examining isovorticity surfaces at different magnitudes is helpful in discerning details in the flow. The reconnection between the $\mathrm{SV}$ and $\mathrm{SV}^{\prime}$ is clearly seen at time $t=64.8, \Omega$ $=1.0$ (Fig. 7). At this time part of the SV has reconnected with its image while the other (vertical) portion, sometimes called a "thread," ${ }^{23}$ remains as it was. The thread is highly stretched by the legs of the SV and quickly disappears, leaving the vortex fully reconnected. Normally the thread bows in the negative $y$-direction due to the influence of the legs but the nearness of the wall prevents this. The bending of the thread was observed in other simulations in which reconnection occurs earlier. This process has been seen in other studies that concentrate on reconnection (see, for example, Melander and Hussain ${ }^{23}$ ). The primary and secondary vortices, however, do not appear to reconnect. The head of the SV is destroyed by dissipation while the PV remains [see Fig.

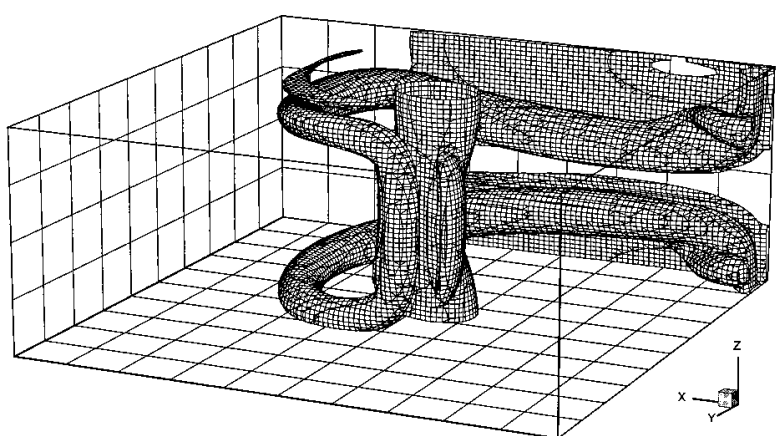

FIG. 7. Surfaces of constant vorticity magnitude $\Omega=1.0$ at $t=64.8$.

6(e)]. Since the SV is weaker than the PV its vorticity in the bridge will dissipate first. If the bridge still contains significant vorticity (due to the PV) then reconnection cannot occur. The primary vortex will continue to stretch the secondary with the resulting increased dissipation hastening its destruction.

An energy cascade based on the previous observations can be described as follows. Initially, if we consider only one member of the pair, there is a single vortex (the primary). As the primary vortex approaches the wall a smaller oppositesigned secondary vortex is created. The secondary vortex is unstable to the short-wavelength Crow-Widnall $(\mathrm{CW})$ instability due to the influence of the primary. As the instability grows the secondary vortex is bent, stretched, and wrapped around the stronger primary. Thus the vorticity of the secondary vortex which was originally in the axial ( $z-)$ direction now lies mostly in the cross plane. The configuration resembles a continuous series of hairpin vortices. The heads, which are short sections of the secondary vortex with axes parallel to the primary's, connect the legs of the secondary vortex. The legs lie primarily in the cross plane and are longer than the heads because of stretching. These legs form a series of counter-rotating vortices in the $z$-direction. As the secondary vortex approaches the wall each leg creates its own vorticity layer on the wall which can roll up to form an even smaller tertiary vortex. The $\mathrm{CW}$ instability can grow in the tertiary vortices as well as they revolve around the legs of the secondary vortex and approach the wall. Since the TV cores are smaller than those of the SV the resulting wavelength of the instability is smaller. Thus energy is transferred to continually finer scales by the creation of smaller and smaller vortices. Dissipation, of course, controls the finest scale of the flow.

\section{The growth of the kinetic energy of the disturbance}

It is desirable to discover the most unstable wave since this is the wave that would naturally dominate under general disturbances. To do this it is necessary to quantify the growth of the instability. This has been done by taking a fast Fourier transform (FFT) in the $z$-direction of the energy at regular intervals in time. The energy $q$ is defined as twice the kinetic energy per unit of mass. On a uniform grid $q=\Sigma_{i, j, k}\left(u^{2}\right.$ $\left.+v^{2}+w^{2}\right)$. In Fig. 8 the FFT coefficients are shown for the first three modes $\left(\hat{q}_{1}, \hat{q}_{2}\right.$, and $\left.\hat{q}_{3}\right)$ as functions of time. Ini- 


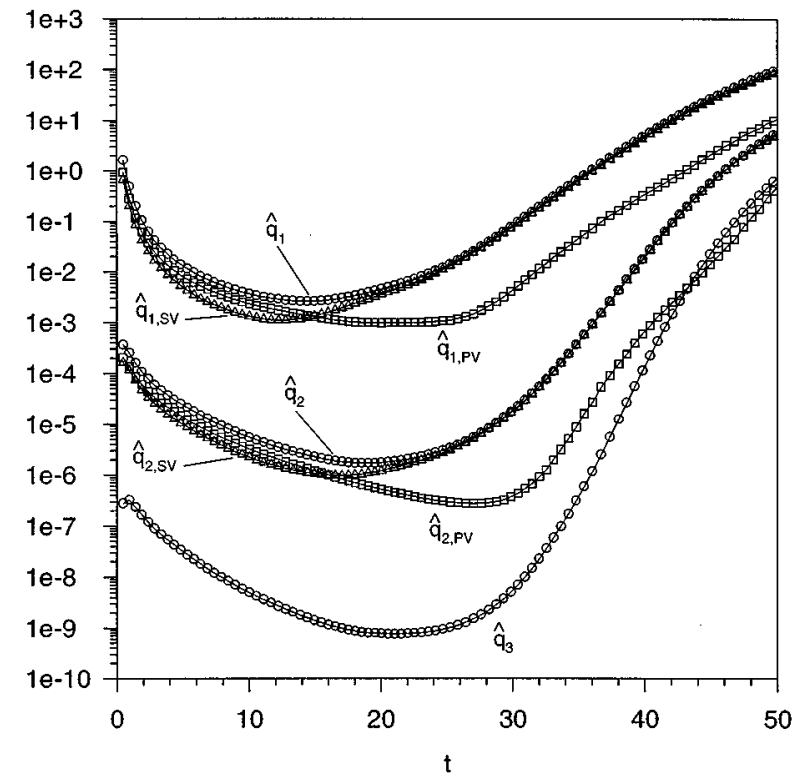

FIG. 8. The temporal development of the kinetic energy in the first three modes.

tially the energy in the first mode decays but it slows as the primary vortex nears the wall. Between times 12.2 and 19.7 the secondary vortex is developing on the wall. During this time the kinetic energy begins to grow. After $t=19.7$ the SV is fully formed and both vortices begin to move away from the wall. The kinetic energy begins to grow exponentially at a rate of 0.41 . The energy of the second mode is also growing exponentially, but at a higher rate $\left(\alpha_{2}=0.76\right)$ while the growth rate for the third mode is even higher $\left(\alpha_{3}=1.17\right)$. By $t=50$ the energy level of the second mode is much closer to that of the first, though it is still an order of magnitude less. Also the growth rates of both modes have begun to decrease, indicating that nonlinear effects are becoming important and a cascade of energy to higher modes has begun. Viscous dissipation is not responsible since the spectrum (not shown here but see Fig. 16 for related results) shows a buildup of energy for high wave numbers. In addition, the SV is nearing the center plane so the effect of the images is becoming important as well.

Also shown in Fig. 8 is the decomposition of the energy for each mode into two components labeled PV and SV. These are an approximation of the energy in the primary and secondary vortices. They are computed as follows. At each point in the cross plane compute the kinetic energy FFT coefficients $\hat{q}_{1}(x, y)$ and $\hat{q}_{2}(x, y)$. If $\omega_{z}\left(x, y, z_{\text {mid }}\right)<0$ (that is, the $z$-component of vorticity at the mid plane has the same sign as that of the $\mathrm{SV}$ ) then the kinetic energies contribute to the secondary vortex components $\hat{q}_{1, \mathrm{SV}}$ and $\hat{q}_{2, \mathrm{SV}}$. Otherwise they contribute to the primary vortex components.

These components should be treated with care. First of all, the SV label is somewhat misleading since the secondary vortex does not exist initially despite the nonzero values of $\hat{q}_{1, \mathrm{SV}}$ for small times. This merely represents the part of the disturbance that happens to give $\omega_{z}<0$ on the horizontal mid plane. Secondly, it is improper to think of the flow as divided into parts corresponding to the two vortices only. There are regions of the flow far from both vortices. However, the energy of the disturbance in these regions is dissipated by viscous effects since there is no mechanism by which it can extract energy from the mean flow. The energy $\hat{q}_{\mathrm{SV}, 1}$ has a component due to the wall vorticity layer but its contribution is small since $\hat{q}_{\mathrm{SV}, 1}$ does not increase until after the secondary vortex forms. The most important consideration is that there is some ambiguity to the relationship between the energy in the secondary vortex and $\hat{q}_{1, \mathrm{~Sv}}$ after the vortex bends significantly. In the region between the primary and secondary vortices $\omega_{z}$ can change sign along a $z$ line. This raises the question of whether the energy for this line should be counted as part of the PV or SV. Consequently these components are approximate and are not valid for large times.

This being said, some useful insights can be gained by this decomposition. Initially $\hat{q}_{1, \mathrm{~Sv}}$ decays to a lower level so that more of the first mode energy in the flow resides in the primary vortex. As the secondary vortex forms $\hat{q}_{1, \mathrm{~Sv}}$ begins to grow rapidly while $\hat{q}_{1, \mathrm{PV}}$ continues to decrease. During this time the first mode SV energy surpasses that of the PV. By the time the secondary vortex is fully formed it contains most of the first mode energy of the flow. Later $\hat{q}_{1, \mathrm{PV}}$ begins to grow but almost all of the first mode energy remains in the secondary vortex. The energy of the second mode behaves in a similar fashion but its value is much less.

In order to determine the wavelength of the most unstable wave a series of ten simulations were performed. Each varies from another only in the value of $L_{z}$. The size of the domain in the cross plane is $L_{x}=10, L_{y}=10$ while $L_{z}$ varies from 1 to 12 . Each case is initialized by a RandB disturbance of the first mode $\left(\lambda_{z}=L_{z}\right)$. The level of disturbance is $U_{\mathrm{rms}}^{\prime}$ $=0.02 v_{c}$. As before, the Reynolds number is $\operatorname{Re}_{\Gamma}=2196$ and the vortex is initially located at $x=2, y=5$. For each case the growth rate of $\hat{q}_{1}$ was determined in the exponential growth period between $t=30$ and $t=37$. The results are shown in Fig. 9 as a function of the wave number. The maximum growth rate is $\alpha_{1}=0.41$ and occurs at $k=1.57\left(\lambda_{z}\right.$ $=4.0$ ). Because the curve is rather flat near the maximum it is difficult to obtain an accurate value for the most amplified $k$. For comparison we can use the theoretical predictions of the Widnall et al. short-wavelength instability of a vortex pair. However, there are some important differences between the Widnall et al. analysis and the current flow simulations. Their analysis assumes that the vortices are identical and the separation distance is large compared to the core diameter. Neither is true here. The secondary vortex is smaller and weaker than the primary vortex and they are very close together. In fact, the cross sectional shape of the vortices, especially for the SV, is distorted. Nevertheless, the work of Widnall et al. is the most appropriate that exists for comparison. Widnall et al. predict that the first radial mode is stable. For the second radial mode the most unstable wave is predicted to have a wavelength of $3.22 r_{c}$. This compares reasonably well with the value of approximately $4 r_{c}$ obtained by the current simulations, especially considering the differences mentioned above and the difficulty of obtaining an accurate value from the simulations. The agreement with the Widnall et al. predictions improves considerably when the 


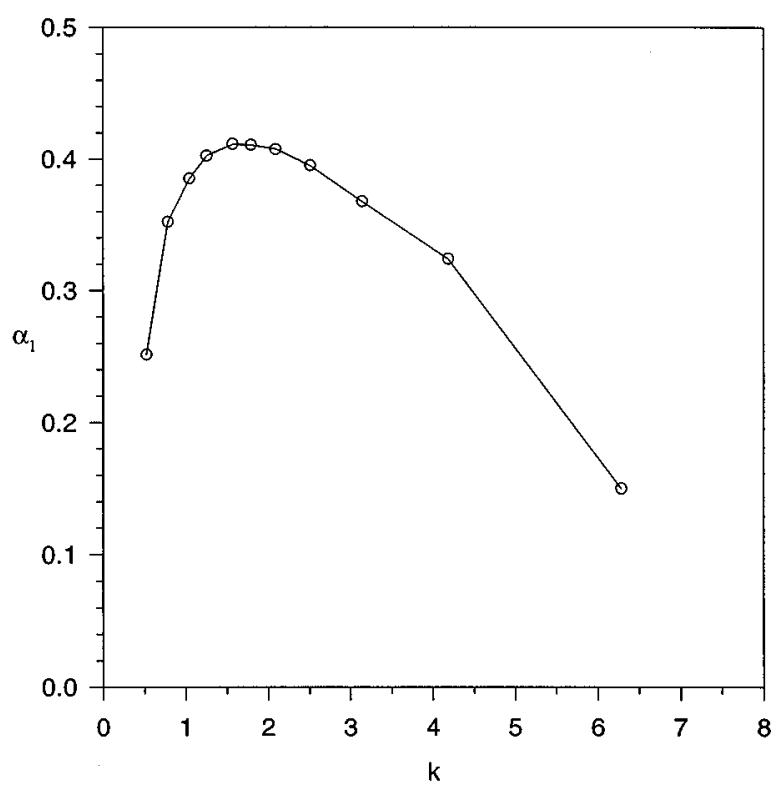

FIG. 9. The growth rate of the first mode of kinetic energy as a function of the wave number.

domain is lengthened in the axial direction (see Section III E).

\section{Grid independence study}

To demonstrate grid independence the simulation described in Section III B was repeated with a grid refined in the cross plane. A uniform grid of size $144 \times 144 \times 48$ was used resulting in 29 grid points in the vortex core. The time step was determined by the Courant-Friedrichs-Lewy condition. For both cases, as well as all simulations described herein, $\mathrm{CFL}=0.5$. This is well below the stability limit of $\mathrm{CFL}=\sqrt{3}$ for the one-dimensional convection-diffusion equation. The time step for the simulation with the $96 \times 96$ $\times 48$ grid is $\Delta t \approx 0.030$ for small times. Figure 10 shows the vorticity distribution on a horizontal plane through the center of the domain for each simulation at $t \approx 64$. The contour levels are identical in each case. The secondary vortex is seen as a concentrated region of negative vorticity appearing above the larger, more distorted primary vortex. Only small changes are evident in the vortices between the simulations. In particular, the size and strength of the SV is not affected by numerical dissipation. On the wall the finer grid simulation shows a stronger and slightly larger vorticity layer. However, it is apparent that the incomplete resolution of the wall vorticity layer does not affect the motion or structure of the vortices. The same observation was noted by Orlandi and Verzicco $^{14}$ who concluded that the complete resolution of the very thin wall vorticity layer is not necessary for accurate computation of the large scales. In addition, surfaces of constant vorticity (not shown here) reveal that the core position of both vortices and the reconnection region are nearly identical to the coarser grid results. For both cases the growth rate of the kinetic energy between times 30 and 37 is 0.42 .

Two more simulations were also run in order to determine the effect of the cross plane domain size. In the first a

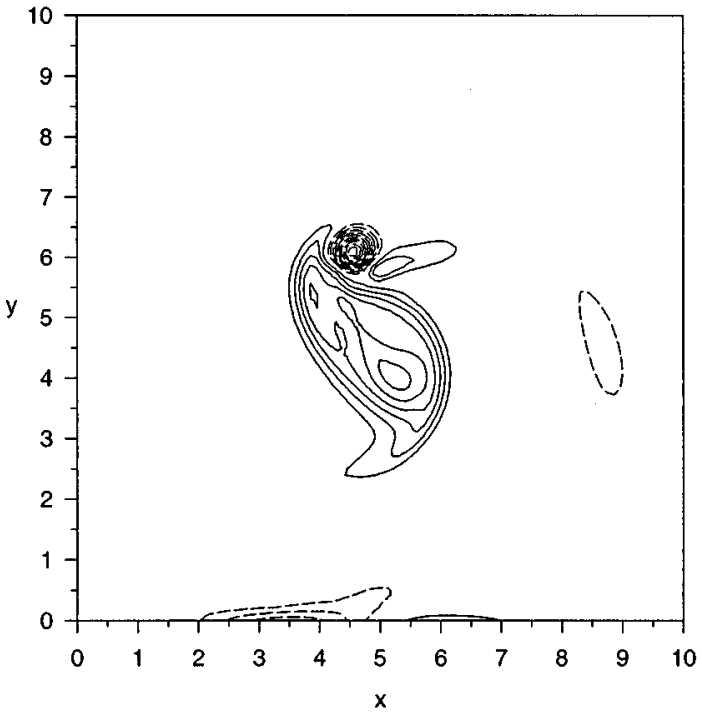

(a)

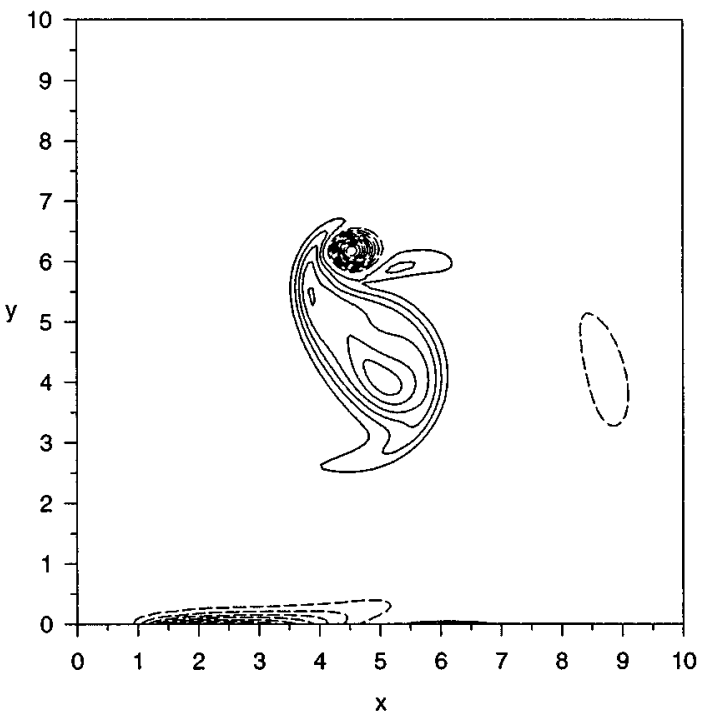

(b)

FIG. 10. Vorticity contours of $\omega_{z}$ on a horizontal plane through the center of the domain at $t \approx 64$. The contour levels range from -3 to 2 by 0.25 . Dashed lines represent negative vorticity while solid lines represent positive vorticity. (a) $96 \times 96 \times 48$ grid; (b) $144 \times 144 \times 48$ grid.

domain size of $L_{x}=L_{y}=10$ and $L_{z}=2.5$ was used with a uniform grid of size $64 \times 64 \times 16$. In the second simulation the length of the domain and the number of points in the $x$ and $y$-directions was doubled. The smaller domain affects the trajectories of the vortices somewhat, shifting them in the negative $x$-direction by approximately half of a core radius and downward slightly. The form of the instability and its growth rate remain unchanged.

\section{E. Unbiased disturbance}

Until now simulations have been presented for cases in which the initial random disturbance consists of only one mode in the $z$-direction. Let us now consider a case in which all modes are seeded by an initial random disturbance. As before the Reynolds number is $\operatorname{Re}_{\Gamma}=2196$. A Rand disturbance of amplitude $U_{\mathrm{rms}}^{\prime}=0.20 v_{c}$ is used so that there is no 
structure to the disturbance in the cross plane. The length of the domain in the axial direction has been increased to $L_{z}$ $=16$ so that longer wavelengths can be seen. A uniform grid of size $64 \times 64 \times 128$ is used which gives 13 grid points in the vortex core. A time series of isovorticity surfaces is shown in Fig. 11. The general features of the flow are similar to the ones of the simulation described in Section III B. At time $t$ $=43.0$ significant perturbations have already appeared. There is clearly more than one wavelength involved. As before the secondary vortex disturbance grows very rapidly and begins to wrap around the PV [Fig. 11(b)]. As the SV becomes highly distorted its diameter increases significantly. The dominant wave here appears to have a wavelength of $3.2 r_{c}$. Unlike the previous case, however, the secondary vortex is not in contact with the symmetry plane so the SV cannot reconnect with its image. At time $t=62.4$ the SV has wrapped even further around the PV. Vorticity stretching has greatly decreased the cross sectional diameter of the SV. The horizontal legs of the secondary vortex have moved in the $z$-direction such that they are grouped in pairs. The mechanism behind this pairing will be explained shortly. As in the previous simulation, the $\mathrm{SV}$ is also generating vorticity at the wall. By time $t=75.2$ the heads of the secondary vortex have been destroyed leaving the legs which are now disorganized. The legs are destroyed by stretching and dissipation, leaving only the now distorted primary vortex [Fig. 11(e)].

The shape of the secondary vortex at $t=62.4$ is more easily seen in Fig. 12 which shows the isovorticity surfaces at $t=62.4$ for a higher vorticity level and a different viewing angle. The structure of the SV in the $z$-direction is quite regular with the exception of a region near the top of the domain where the vortex is less organized. The vertical "heads" of the secondary vortex can just be seen immediately to the left of the primary. Unlike the previous simulation, the secondary vortex does not reconnect with its image across the center plane but rather forms a series of "hairpin" bends. Whether or not the SV reconnects with its image depends in part on the characteristics of the initial disturbance. As the SV moves between the PV and the symmetry plane, reconnection can occur only if the instability has grown sufficiently such that parts of the SV are in close contact with the symmetry plane. Otherwise, reconnection cannot occur. Another consideration is the effect of the symmetry assumption. Physically the flow could develop asymmetries which are not permitted in these simulations. The asymmetries could produce significant changes in the flowfield, particularly for reconnection. Yet regardless of whether reconnection occurs the other general features of the flow such as the hairpin shape of the secondary vortex and the generation of vorticity on the wall due to the secondary vortex remain unchanged.

Plotting the pressure and vorticity on various planes can also yield valuable insights. Let us first examine the pressure on the wall at $t=62.4$ as shown in Fig. 13. There are five high pressure regions. The high pressure is due to the legs of the secondary vortex forcing fluid toward the wall. The low pressure regions correspond to the areas where the legs move fluid away from the wall. This can be clearly seen by examining the contours of $\omega_{x}$ on the plane $x=2.97$ which cuts through the high pressure regions (see Fig. 14). The legs of the SV appear as a series of counter-rotating vortices. They were originally uniformly spaced in $z$ but have now grouped into pairs. This is a direct result of the influence of the images across the wall. Each positive vortex (the upper member of each pair) has a negative image which induces a downward motion. The opposite is true for the negative vortices. Thus the vortices are grouped such that the flow between them is away from the wall while the flow between the pairs is toward the wall. By comparing Figs. 13 and 14 it can be seen that the high pressure areas on the wall lie in the "stagnation" region between the vortex pairs.

Let us also consider a vertical plane at $y=3.44$ which passes through the primary vortex center (see Fig. 15). The vorticity magnitude is plotted in order that both the primary and secondary vortices are visible. Once again the legs of the SV have paired but a comparison with Fig. 14 shows that the pairs are offset from the ones near the wall. Two legs that are close together at the PV are far apart at the wall. Between the members of each pair fluid is pumped away from the primary vortex. Between the pairs fluid is moved toward the PV. This appears to be the primary mechanism by which the PV is bent. The core of the primary vortex has also developed axial variations in the pressure which leads to a periodic axial velocity along its core.

Identifying the important wavelengths of the flow can be accomplished by applying a FFT in the $z$-direction. This has been done for the total kinetic energy at nine different times. The spectra at these times are presented in Fig. 16. It is evident that the flow is well resolved since the spectrum at the small scales shows the characteristic slope of -7 for dissipation (a consequence of Heisenberg's hypothesis ${ }^{34}$ ). By time $t=20.5$ the spectrum has begun to show variations. Peaks emerge at the fifth and ninth modes. This time is after the SV forms but well before it interacts with its image or the wall. Between times 20.5 and 53.4 the energy grows rapidly, while the fundamental shape of the spectrum does not change. In this time period the secondary vortex disturbance becomes large [see Fig. 11(b)]. After time 53.4 the energy decays slowly. By the end of the simulation the spectrum is quite smooth. The peaks have disappeared and the energy at the smaller scales has decreased appreciably.

The peak for the fifth mode $\left(\lambda_{z}=3.20 r_{c}\right)$ corresponds to the second radial mode of the short-wavelength instability seen earlier (see Fig. 9). For a vortex with a vorticity distribution of $\left(r^{2}-r_{c}^{2} / 4\right)^{2}$ Widnall et al. found the wavelength of the most amplified wave to be $3.22 r_{c}$. The agreement is excellent. According to Widnall et al. the third radial mode has a wavelength of $1.80 r_{c}$ for the most unstable wave. This also agrees closely with the current simulation which predicts the second peak to occur at the ninth mode $\left(\lambda_{z}\right.$ $=1.78 r_{c}$ ). These results confirm that the instability of the secondary vortex is the short-wavelength $\mathrm{CW}$ instability. To the authors' knowledge, this is the first three-dimensional simulation that lends support to the short-wavelength instability on interacting vortices. It has been shown how this instability leads to three dimensionality after the rebound of a vortex pair. 


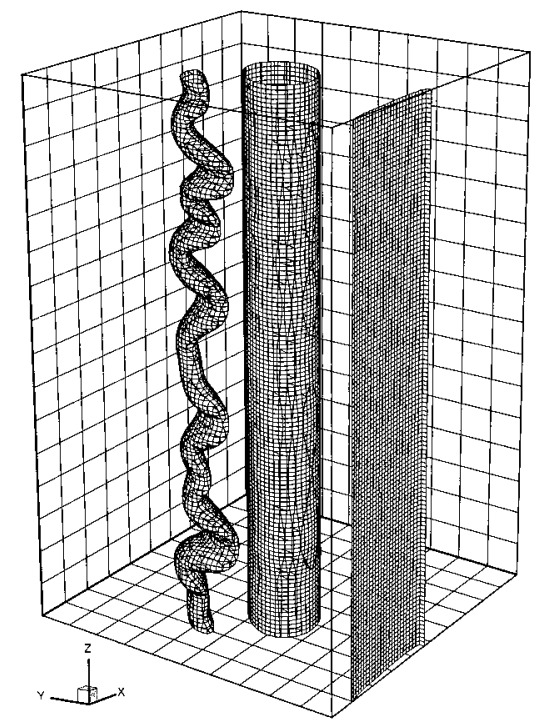

(a)

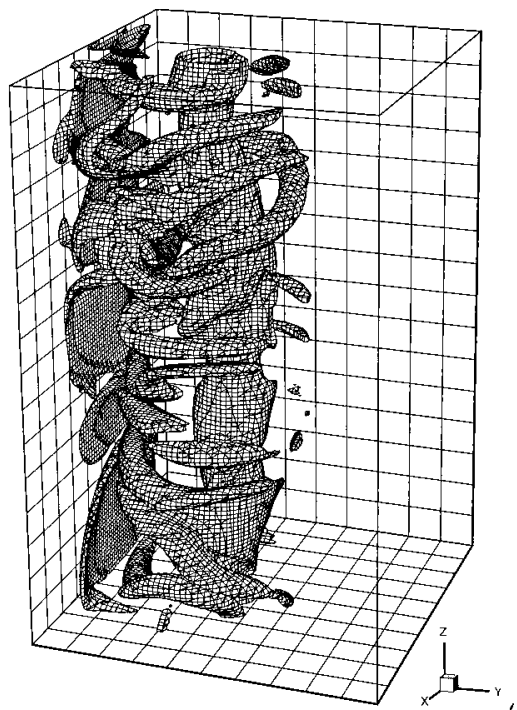

(d)
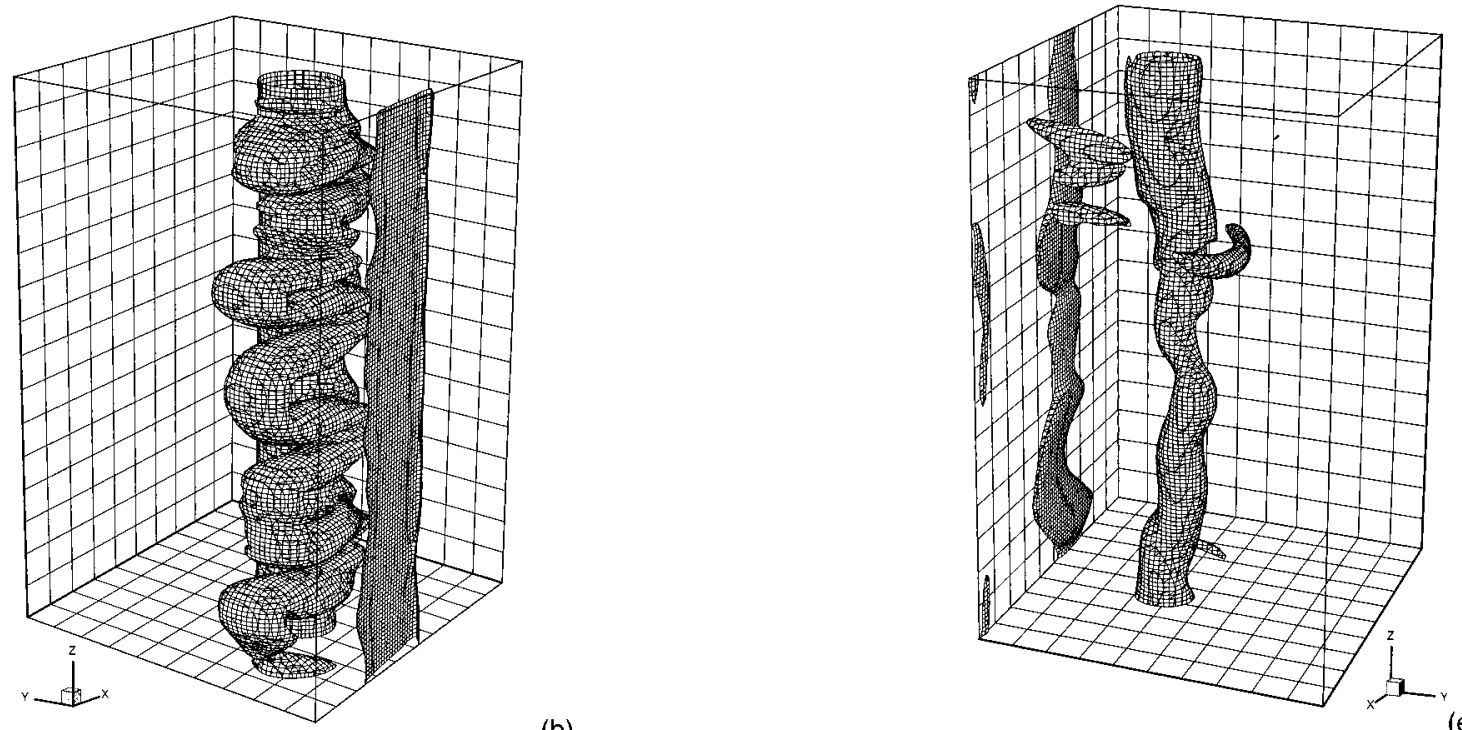

(e)

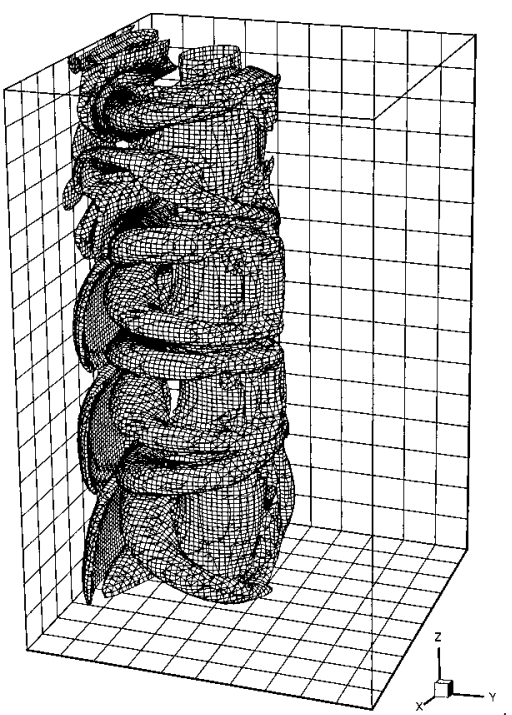

(c)

FIG. 11. Surfaces of constant vorticity magnitude $\Omega=0.7$. (a) $t=43.0$, (b) $t=53.4$, (c) $t=62.4$, (d) $t=75.2$, (e) $t=109.4$.

dimensionality is introduced into the initially twodimensional flow. This mechanism is a short-wavelength Crow-Widnall instability of the secondary vortex. To the authors' knowledge, this is the first three-dimensional simu- 


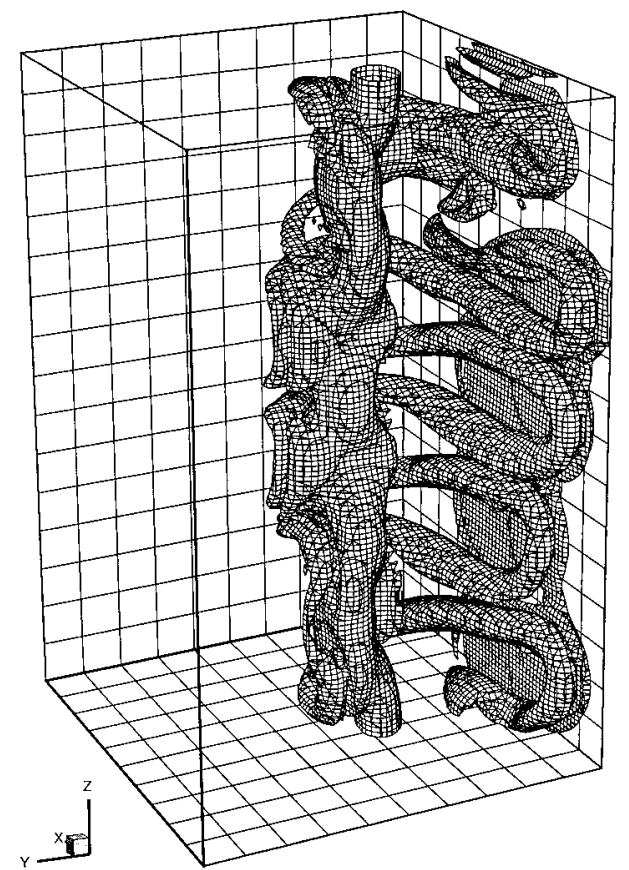

FIG. 12. Surfaces of constant vorticity magnitude $\Omega=1.0$ at $t=62.4$.

lation to reveal this instability of the secondary vortex. The most preferred wavelength of the secondary vortex was found to be $3.20 r_{c}$. This value agrees extremely well with the analysis of Widnall et al. ${ }^{18}$ which predicts $\lambda_{z}=3.22 r_{c}$ for the second radial mode. In the current simulations a higher

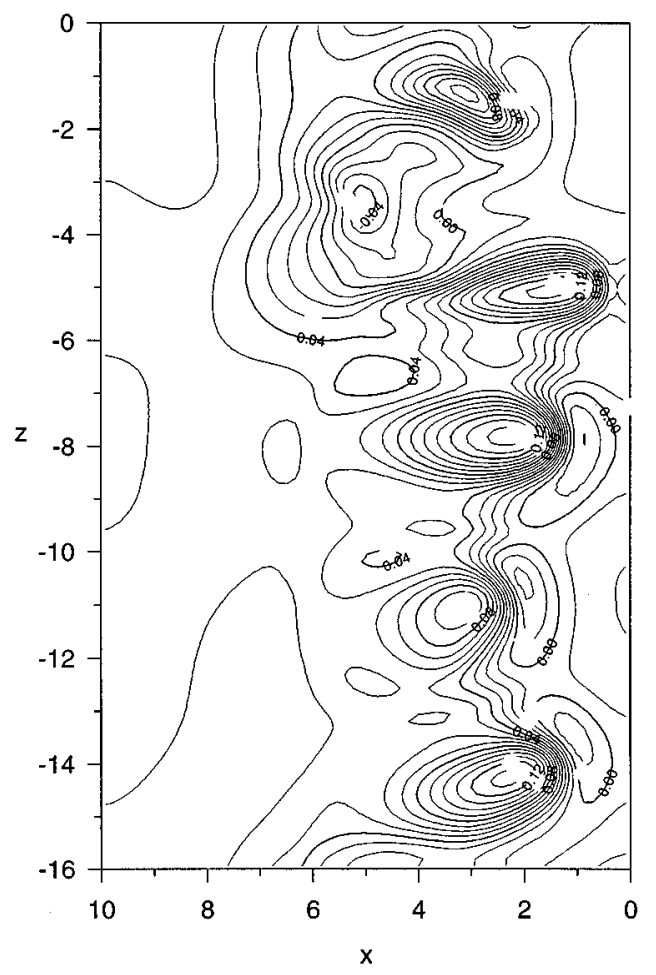

FIG. 13. The pressure on the wall at $t=62.4$.

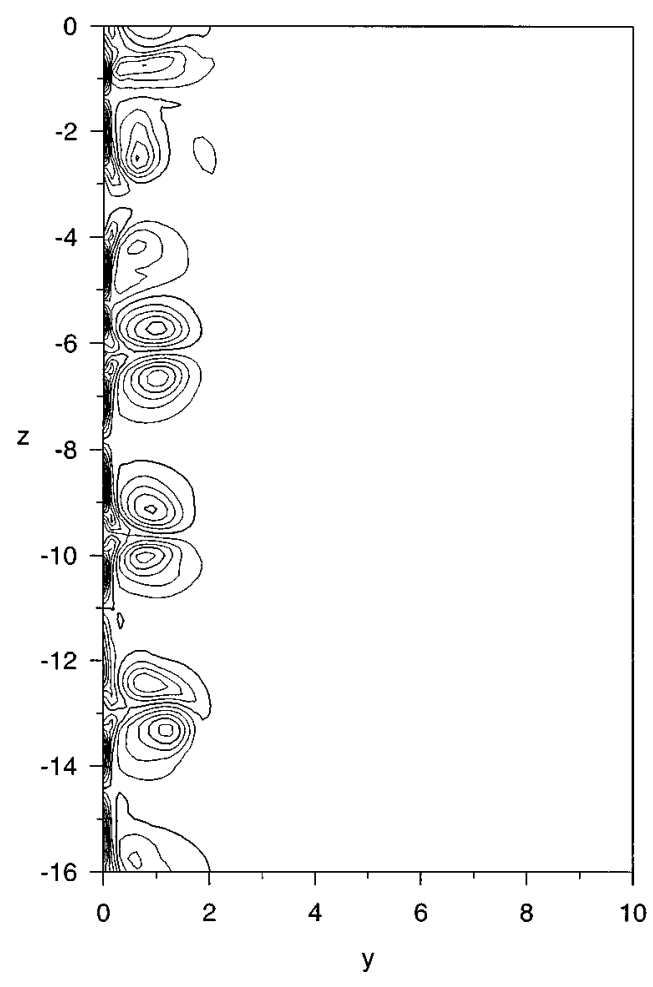

FIG. 14. Contours of $\omega_{x}$ on the plane $x=2.97$ at $t=62.4$. The contour levels range from -6 to 6 by 0.5 .

mode of wavelength $1.78 r_{c}$ was also seen. Its wavelength compares very well with the one for the third radial mode of Widnall et al. $\left(\lambda_{z}=1.80 r_{c}\right)$. These waves were found to emerge naturally from an unbiased random disturbance.

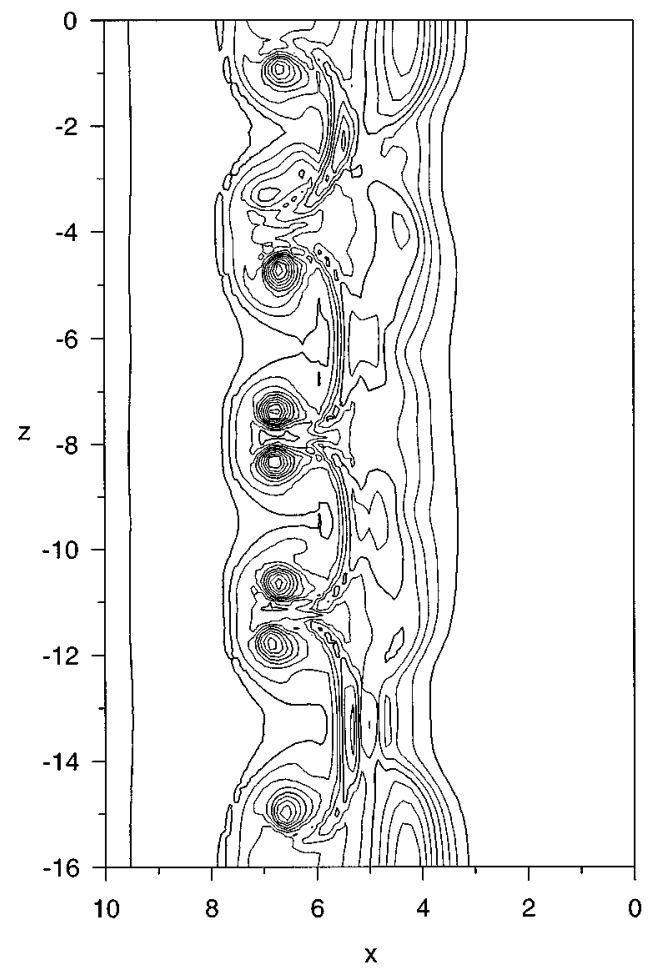

FIG. 15. Contours of vorticity magnitude on a vertical plane through the primary vortex $(y=3.44)$ at $t=62.4$. The contour levels range from -3 to 3 by 0.25 . 


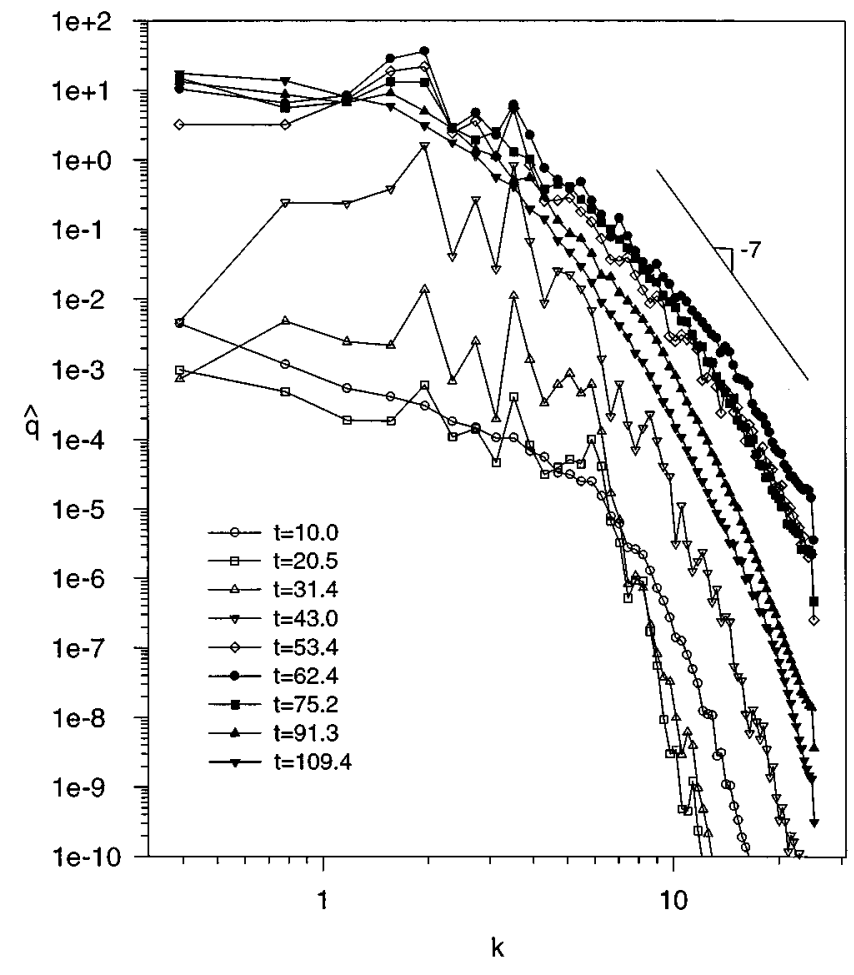

FIG. 16. The kinetic energy spectrum.

The growth of the instability of the secondary vortex in the presence of the stronger primary leads to the turning and intense stretching of the secondary. The undulations of the secondary vortex were observed to reconnect with its image at the center plane, thus forming a series of elongated loops. Reconnection does not occur between the primary and secondary vortices. The secondary vortex is destroyed by stretching and dissipation leaving the primary vortex with a permanently distorted shape but relatively unaffected strength compared to an isolated vortex.

A new type of energy cascade based on the growth of the Crow-Widnall instability has been proposed. It can be described as follows. As the primary vortex initially approaches the wall a smaller opposite-signed secondary vortex is created. The secondary vortex is unstable to the shortwavelength Crow-Widnall instability. As the instability grows the secondary vortex is bent, stretched, and wrapped around the stronger primary. Thus the vorticity of the secondary vortex which was originally in the axial ( $z$-) direction now lies mostly in the cross plane. The configuration resembles a continuous series of hairpin vortices. The legs, which are the longer sections of the secondary vortex lying primarily in the cross plane, form a series of counter-rotating vortices in the $z$-direction. As the secondary vortex approaches the wall each leg creates its own vorticity layer on the wall which can roll up to form an even smaller tertiary vortex. The Crow-Widnall instability can grow in the tertiary vortices as well as they revolve around the legs of the secondary vortex and approach the wall. Since the cores are smaller than those of the secondary vortex the resulting wavelength of the instability is smaller. Thus energy is trans- ferred to continually finer scales by the creation of smaller and smaller vortices.

\section{ACKNOWLEDGMENT}

This work is supported by the Office of Naval Research under Grant No. N00014-92-J-4087.

${ }^{1}$ Z. C. Zheng and R. L. Ash, "Study of aircraft wake vortex behavior near the ground," AIAA J. 34, 580 (1996).

${ }^{2}$ D. K. Tafti and S. P. Vanka, "A numerical study of flow separation and reattachment on a blunt plate," Phys. Fluids A 3, 1749 (1991).

${ }^{3}$ J. A. Luton, S. A. Ragab, and D. P. Telionis, "Interaction of spanwise vortices with a boundary layer," Phys. Fluids 7, 2757 (1995).

${ }^{4}$ C. Koromilas and D. P. Telionis, "Unsteady laminar separation-an experimental study," J. Fluid Mech. 97, 347 (1980).

${ }^{5}$ E. R. Booth, Jr. and Y. C. Yu, "Two-dimensional blade-vortex visualization investigation," AIAA J. 24, 1468 (1986).

${ }^{6}$ T. L. Doligalski, C. R. Smith, and J. D. A. Walker, "Vortex interactions with walls,' Annu. Rev. Fluid Mech. 26, 573 (1994).

${ }^{7}$ F. S. Dee and O. P. Nicholas, "Flight measurement of wing tip vortex motion near the ground," CP 1065, British Aeronautical Research Council, 1968

${ }^{8}$ J. K. Harvey and F. J. Perry, "Flowfield produced by trailing vortices in the vicinity of the ground," AIAA J. 9, 1659 (1971).

${ }^{9} \mathrm{U}$. Boldes and J. C. Ferreri, "Behavior of vortex rings in the vicinity of a wall," Phys. Fluids 16, 2005 (1973).

${ }^{10}$ A. J. Peace and N. Riley, "A viscous vortex pair in ground effect," J. Fluid Mech. 129, 409 (1983).

${ }^{11}$ J. D. A. Walker, C. R. Smith, A. W. Cerra, and T. L. Doligalski, "The impact of a vortex ring on a wall," J. Fluid Mech. 181, 99 (1987).

${ }^{12}$ A. W. Cerra, Jr. and C. R. Smith, "Experimental observations of vortex ring interaction with the fluid adjacent to a surface," Report FM-4, Department of Mechanical Engineering and Mechanics, Lehigh University, Bethlehem, PA, 1983.

${ }^{13}$ P. Orlandi, "Vortex dipole rebound from a wall,"' Phys. Fluids A 2, 1429 (1990).

${ }^{14}$ P. Orlandi and R. Verzicco, "Vortex rings impinging on walls: Axisymmetric and three-dimensional simulations," J. Fluid Mech. 256, 615 (1993).

${ }^{15}$ J. D. Swearingen, J. D. Crouch, and R. A. Handler, "Dynamic and stability of a vortex ring impacting a solid boundary," J. Fluid Mech. 297, 1 (1995).

${ }^{16}$ S. E. Widnall and J. P. Sullivan, "On the stability of vortex rings," Proc. R. Soc. London, Ser. A 332, 335 (1973).

${ }^{17}$ S. C. Crow, "Stability theory for a pair of trailing vortices," AIAA J. 8, $2172(1970)$.

${ }^{18}$ S. E. Widnall, D. B. Bliss, and C. Y. Tsai, "The instability of short waves on a vortex ring," J. Fluid Mech. 66, 35 (1974).

${ }^{19}$ P. J. Thomas and D. Auerbach, "The observation of the simultaneous development of a long- and short-wave instability mode on a vortex pair," J. Fluid Mech. 265, 289 (1994)

${ }^{20}$ W. T. Ashurst and D. I. Meiron, "Numerical study of vortex reconnection," Phys. Rev. Lett. 58, 1632 (1987).

${ }^{21}$ S. Kida, M. Takaoka, and F. Hussain, "Collision of two vortex rings," J. Fluid Mech. 230, 583 (1991).

${ }^{22}$ M. J. Shelley, D. I. Meiron, and S. A. Orszag, "Dynamical aspects of vortex reconnection of perturbed anti-parallel vortex tubes," J. Fluid Mech. 246, 613 (1993).

${ }^{23} \mathrm{M}$. V. Melander and F. Hussain, "Cross-linking of two antiparallel vortex tubes," Phys. Fluids A 1, 633 (1989).

${ }^{24}$ S. Kida and M. Takaoka, "Vortex reconnection," Annu. Rev. Fluid Mech. 26, 169 (1994).

${ }^{25}$ D. G. Dommermuth, "The formation of U-shaped vortices on vortex tubes impinging on a wall with applications to free surfaces,' Phys. Fluids A 4, 757 (1992).

${ }^{26}$ D. G. Dommermuth, "The laminar interactions of a pair of vortex tubes with a free surface,'” J. Fluid Mech. 246, 91 (1993).

${ }^{27}$ T. Sarpkaya and P. Suthon, "Interaction of a vortex couple with a free surface,' Exp. Fluids 11, 205 (1991).

${ }^{28}$ T. Sarpkaya, "Three-dimensional interactions of vortices with a free surface," AIAA Paper No. 92-0059, 1992. 
${ }^{29}$ J. Kim and P. Moin, "Application of a fractional-step method to incompressible Navier-Stokes equation,'” Comput. Phys. 59, 308 (1985).

${ }^{30} \mathrm{H}$. Le and P. Moin, "An improvement of fractional-step methods for the incompressible Navier-Stokes equations," J. Comput. Phys. 92, 369 (1991).

${ }^{31}$ J. A. Luton, "Numerical simulations of vortices near free and solid surfaces," Ph.D. dissertation, Virginia Polytechnic Institute and State Uni- versity, Department of Engineering Science and Mechanics, Blacksburg, VA, 1996.

${ }^{32}$ A. J. Bilanin and S. E. Widnall, "Aircraft wake dissipation by sinusoidal instability and vortex breakdown," AIAA Paper No. 73-107, 1973.

${ }^{33} \mathrm{H}$. Chevalier, "Flight test studies of the formation and dissipation of trailing vortices," J. Aircr. 10, 14 (1973).

${ }^{34}$ J. O. Hinze, Turbulence (McGraw-Hill, New York, 1975). 